\title{
Gluten-induced symptoms in diarrhea-predominant irritable bowel syndrome are associated with increased myosin light chain kinase activity and claudin-15 expression
}

\author{
Richard L Wu ${ }^{1,2}$, Maria I Vazquez-Roque ${ }^{3}$, Paula Carlson ${ }^{3}$, Duane Burton ${ }^{3}$, Madhusudan Grover ${ }^{3}$, Michael Camilleri ${ }^{3}$ and \\ Jerrold R Turner,
}

The mechanisms underlying diarrhea-predominant irritable bowel syndrome (IBS-D) are poorly understood, but increased intestinal permeability is thought to contribute to symptoms. A recent clinical trial of gluten-free diet (GFD) demonstrated symptomatic improvement, relative to gluten-containing diet (GCD), which was associated with reduced intestinal permeability in non-celiac disease IBS-D patients. The aim of this study was to characterize intestinal epithelial tight junction composition in IBS-D before and after dietary gluten challenge. Biopsies from 27 IBS-D patients (13 GFD and 14 GCD) were examined by H\&E staining and semiquantitative immunohistochemistry for phosphorylated myosin II regulatory light chain (MLC), MLC kinase, claudin-2, claudin-8 and claudin-15. Diet-induced changes were assessed and correlated with urinary mannitol excretion (after oral administration). In the small intestine, epithelial MLC phosphorylation was increased or decreased by GCD or GFD, respectively, and this correlated with increased intestinal permeability $(P<0.03)$. Colonocyte expression of the paracellular $\mathrm{Na}^{+}$channel claudin-15 was also markedly augmented following GCD challenge $(P<0.05)$. Conversely, colonic claudin-2 expression correlated with reduced intestinal permeability $(P<0.03)$. Claudin-8 expression was not affected by dietary challenge. These data show that alterations in MLC phosphorylation and claudin-15 and claudin-2 expression are associated with gluten-induced symptomatology and intestinal permeability changes in IBS-D. The results provide new insight into IBS-D mechanisms and can explain permeability responses to gluten challenge in these patients.

Laboratory Investigation (2017) 97, 14-23; doi:10.1038/labinvest.2016.118; published online 21 November 2016

Irritable bowel syndrome (IBS) is a chronic functional gastrointestinal disorder characterized by abdominal discomfort and altered stool form or frequency. ${ }^{1}$ The overall incidence of IBS in western populations is estimated to be $20 \%$ and appears to be increasing. ${ }^{2,3}$ Therapeutic options are limited, particularly for patients with diarrhea-predominant IBS (IBS-D), which, in part, reflects our limited understanding of IBS pathogenesis. ${ }^{4}$ It is likely, however, that multiple mechanisms, including accelerated colonic transit, increased ion secretion, inadequate absorption, low-level immune activation and increased intestinal permeability, all contribute to loose stools in IBS-D. ${ }^{4-6}$
Several factors, including variable symptomatology and disease duration, have made it difficult to interpret results from studies comparing normal subjects to IBS-D patients. For example, it is impossible to know if differences between normal subjects and patients reflect primary pathogenic mechanisms, secondary changes that contribute to disease, or adaptive, that is, homeostatic, compensatory responses that may modify disease severity. This inability to differentiate between adaptive, maladaptive, and pathogenic changes in IBS-D is a major obstacle to identification of effective therapies.

We took advantage of mucosal biopsies collected during a previous clinical trial that randomized IBS-D patients for

${ }^{1}$ Department of Pathology, The University of Chicago, Chicago, IL, USA; ${ }^{2}$ Department of Pathology and Laboratory Medicine, Jackson Memorial Hospital, University of Miami, Miami, FL, USA; ${ }^{3}$ Division of Gastroenterology and Hepatology, Clinical Enteric Neuroscience Translational and Epidemiological Research, College of Medicine, Mayo Clinic, Rochester, MN, USA and ${ }^{4}$ Departments of Pathology and Medicine (GI), Brigham and Women's Hospital and Harvard Medical School, Boston, MA, USA

Correspondence: Professor JR Turner, MD, PhD, Departments of Pathology and Medicine (Gl), Brigham and Women's Hospital and Harvard Medical School, 20 Shattuck Street, TH1428, Boston, MA 02115, USA.

E-mail: jrturner@bwh.harvard.edu

Received 23 August 2016; revised 26 September 2016; accepted 1 October 2016 
challenge with gluten-free diet (GFD) or gluten-containing diet (GCD). ${ }^{5}$ GFD was an effective therapy, as it reduced both numbers of bowel movements and intestinal permeability, as assessed by urinary mannitol recovery, relative to subjects challenged with GCD. Duodenal and rectosigmoid mucosal biopsies were collected in a subset of patients, and small intestinal permeability was measured in each patient before and after the dietary intervention. This approach obviated uncertainties associated with comparisons between patients and healthy subjects and also controlled for inter-individual variation by allowing each patient to be their own reference.

While the previous study was highly informative, it did not comprehensively evaluate molecular aspects of barrier regulation in the biopsies obtained. Specifically, the previous study assessed ZO-1, occludin and claudin-1 mRNA expression in small bowel and colon biopsies and identified increases in colonic, but not small bowel RNA expression. This was not, however, confirmed by immunohistochemistry in the case of ZO-1, and neither immunohistochemistry nor other measures of protein expression were used to determine if changes in occludin and claudin-1 mRNA expression reflected changes in protein expression. Further, the relevance of in vivo occludin downregulation is controversial. ${ }^{7-9}$ Finally, although correlative studies have been reported, no published data have assessed the impact of ZO-1 or claudin-1 downregulation on intestinal barrier function in vivo, making it difficult to understand changes previously reported in IBS.

Here, we focused on three claudin proteins known to be important to intestinal physiology and barrier function, claudins 2, 8 and 15, as well as the myosin light chain kinase (MLCK)-myosin II regulatory light chain (MLC) pathway. Each of these has been demonstrated to regulate paracellular permeability in response to physiological and pathophysiological stimuli. ${ }^{10-15}$ Our data show that small intestinal epithelial MLCK activity, measured as MLC phosphorylation, was upregulated in response to GCD challenge and, conversely, downregulated in response to GFD. Neither expression nor localization of claudins 2,8 or 15 within small intestinal epithelia were affected by dietary challenge. Within colonic epithelia, the paracellular $\mathrm{Na}^{+}$channel claudin-15 was markedly upregulated following GCD challenge, but MLCK expression and activity were unchanged and neither claudin-2 nor -8 expression were affected. These data suggest that the MLCK-MLC pathway, which has been shown to regulate tight junction permeability in response to both physiological and pathophysiological stimuli, contributes to IBS-D symptomatology. Changes in claudin-15 expression can be interpreted as adaptive based on the recognition that paracellular $\mathrm{Na}^{+}$efflux into the lumen supports ongoing transepithelial $\mathrm{Na}^{+}$and nutrient absorption, ${ }^{12,16}$ which in turn provides a driving force for water absorption. ${ }^{17}$

\section{MATERIALS AND METHODS Study Design and Demographics}

This study was conducted using biopsies obtained during a double-blind, randomized controlled clinical trial of GFD in IBS-D. ${ }^{18}$ The inclusion criteria required that all participants had symptoms consistent with Rome II criteria, which were confirmed using a validated questionnaire; that psychological health was intact, as assessed using hospital anxiety and depression inventory; and that all participants were ingesting the gluten before participation in the study. Exclusion criteria were: evidence of celiac disease based on evaluation of biopsies or serologies; prior history of a positive response to gluten restriction; gluten exclusion before the start of the study; use of tobacco products within the previous 6 months; NSAID or aspirin use within the previous week; and use of medications that affect gastrointestinal motility or increase the risk of gastrointestinal bleeding within the previous 2 days.

In the previous study, 28 patients, who were equally randomized to the GFD or GCD, underwent duodenal and colonic biopsies before and after the 4-week dietary intervention. The randomization sequence was generated by computer and including matching the participants on criteria including age, gender and body mass index. One set of post-intervention biopsies for a subject who received GFD was inadequate, leaving a total of 27 subjects (13 GFD and 14 GCD) for analysis. All subjects also underwent intestinal permeability testing, based on urinary recovery of orally administered mannitol between 2 and $8 \mathrm{~h}$ after ingestion, ${ }^{18,19}$ All investigators were blinded to individual patient allocation until data collection was complete. The study was approved by Mayo Clinic Institutional Review Board and registered at Clinicaltrials.gov, NCT01094041.

This investigation takes advantage of permeability and clinical data, as well as biopsies collected from the 55 patients during the prior study. Immunohistochemical analyses reported here were performed following the previous report based on increased understanding of the mechanisms underlying intestinal barrier regulation.

\section{Immunohistochemistry}

Immunohistochemical staining was performed on pre-dietary and post-dietary intervention specimens from small bowel and colon. Briefly, following de-paraffinization samples were rehydrated and antigen was retrieved by boiling in $\mathrm{pH} 6.0$ retrieval solution (Dako, Denmark) for $20 \mathrm{~min}$. Endogenous peroxidase was quenched by immersion in $3 \% \mathrm{H}_{2} \mathrm{O}_{2}$ methanol solution, and nonspecific binding sites were blocked using 5\% goat serum in PBS solution with $0.025 \%$ Triton X-100 (Sigma, St Louis, MO, USA). Primary antibodies dilutions were determined empirically in preliminary experiments. For these specimens, antibodies and concentrations were: rabbit polyclonal anti-claudin-2 (ab53032, $20 \mu \mathrm{g} / \mathrm{ml}$, Abcam, Cambridge, MA, USA), rabbit polyclonal anti-claudin-8 (NBP1-59157, $10 \mu \mathrm{g} / \mathrm{ml}$, Novus 
Biologicals, Littleton, CO, USA), rabbit polyclonal anticlaudin-15 (NBP2-13842, $10 \mu \mathrm{g} / \mathrm{ml}$, Novus Biologicals), mouse monoclonal anti-MLCK (clone K36, $10 \mu \mathrm{g} / \mathrm{ml}$, Sigma), mouse monoclonal anti-Ser19-phosphorylated myosin light chain MLC $(3675,2 \mu \mathrm{g} / \mathrm{ml}$, Cell Signaling, Danvers, MA, USA). After incubation overnight at $4^{\circ} \mathrm{C}$, slides were washed with $0.05 \%$ Tween-20 in TBS. HRP-conjugated, mouse- and rabbit-specific secondary antibodies were included in EnVision kits (Dako), which were also used for final detection. Staining conditions were optimized and validated using human and mouse control tissues with known changes in protein expression or, in the case of myosin light chain, phosphorylation, as previously described. ${ }^{20,21}$ In addition, internal controls, as well as positive and negative controls were included with each staining run.

\section{Microscopic Evaluation}

H\&E-stained slides were evaluated by a subspecialty-trained, gastrointestinal surgical pathologist (JRT) to exclude patients with non-IBS diagnoses. Immunohistochemical stain intensity was graded on a six-point scale based on intensity, with 0 indicating no staining and 6 indicating maximal stain. Scores from at least three separate foci per biopsy were averaged to obtain final scores for each case. The scoring system and staining technique were validated using specimens with known changes in protein expression or myosin light chain phosphorylation. Slides were reviewed in a blinded manner, with a subset reviewed independently by a second observer to validate reproducibility.

All biopsy specimens evaluated included full-length villus (duodenum) or intact surface (colon) epithelium with foci of well-oriented villi and crypts that were suitable for blinded, semiquantitative evaluation. For each biopsy, five separate 40X fields were examined and scored independently using a six-point scale. The final score for each biopsy represented the aggregate median score.

\section{Statistical Analysis}

For MLCK, phosphorylated myosin light chain, claudin-2, claudin-8 and claudin-15 staining the Mann-Whitney rank sum test was used after verifying that the scores were normally distributed. The unpaired $t$-test was used to analyze mannitol excretion between GFD and GCD groups before and after diet challenge. For correlation analysis, the Spearman correlation coefficient (Rho) was determined using the difference between pre- and post-challenge staining scores and the difference between pre- and post-challenge mannitol excretion, that is, urinary recovery, in each subject.

\section{RESULTS}

\section{MLCK and MLC Phosphorylation}

Phosphorylated MLC and MLCK expression were detected primarily in the subapical cytoplasm just beneath the brush border, and at lateral membranes, consistent with previous work. ${ }^{20,22}$ As reported previously, MLCK expression and
MLC phosphorylation were prominent in villus enterocytes and surface colonocytes, relative to crypt epithelia (Figure 1a, Supplementary Figures 1 and 2). ${ }^{23}$ Also consistent with previous studies in inflammatory bowel disease (IBD) and mouse models, changes in MLCK expression and MLC phosphorylation were prominent in villus enterocytes and surface colonocytes. ${ }^{20,24}$ It is, therefore, not surprising that MLCK expression and MLC phosphorylation were limited in crypt epithelium and not different between groups in this study. Our analyses were thus focused on villus and surface enterocytes and colonocytes, respectively. Overall expression of MLCK before dietary challenge was similar in GFD and GCD groups, although there was significant heterogeneity within the overall population.

One exceptional feature of this study was that all subjects were IBS-D patients. This removed baseline differences between normal subjects and IBS-D patients from the analysis, and therefore eliminated the possibility that any observed differences reflected secondary changes that were not specific for IBS-D. Nevertheless, when changes in protein expression and MLC phosphorylation were assessed as group data, similar to previous studies comparing IBS-D patients and healthy controls, effects were limited and were not significantly different between GFD and GCD groups for either small bowel or colon biopsies.

Importantly, this study design allowed each subject to serve as their own control, thereby limiting the impact of interindividual variation due to unrelated factors. When data were re-analyzed based on the changes in MLC phosphorylation before and after dietary challenge, it became clear that small intestinal epithelial MLC phosphorylation was significantly decreased after GFD and, conversely, increased after GCD (Figure 1b; $P=0.05$ ). Morphologically, the increases in MLC phosphorylation were most recognizable as enhanced staining at lateral membranes and within the perijunctional actomyosin ring. Notably, these changes were more subtle than those previously reported in association with active $\mathrm{IBD},{ }^{20}$ consistent with the more limited changes in intestinal permeability reported in IBS- $\mathrm{D}^{19,25}$ relative to IBD. Colonic epithelial MLCK expression and MLC phosphorylation were unaffected by dietary intervention.

\section{Claudins}

Claudins are a large family of tetra-spanning tight junction proteins. Some claudins, for example, claudin-2 and claudin15, are known to form paracellular channels that allow transepithelial flux of ions and small solutes. ${ }^{26-29}$ Expression of other claudins, for example, claudin-4 and claudin-8, reduces paracellular permeability, suggesting that they form the paracellular seal or, alternatively, regulate function of channel-forming claudins. ${ }^{30-35}$ Although changes in claudin4 expression have not been associated with disease, ${ }^{35,36}$ increased claudin-2 expression ${ }^{21,35-38}$ and reduced claudin-8 ${ }^{36}$ expression have been reported in human and experimental IBD. We therefore assessed claudin-2 and 
a

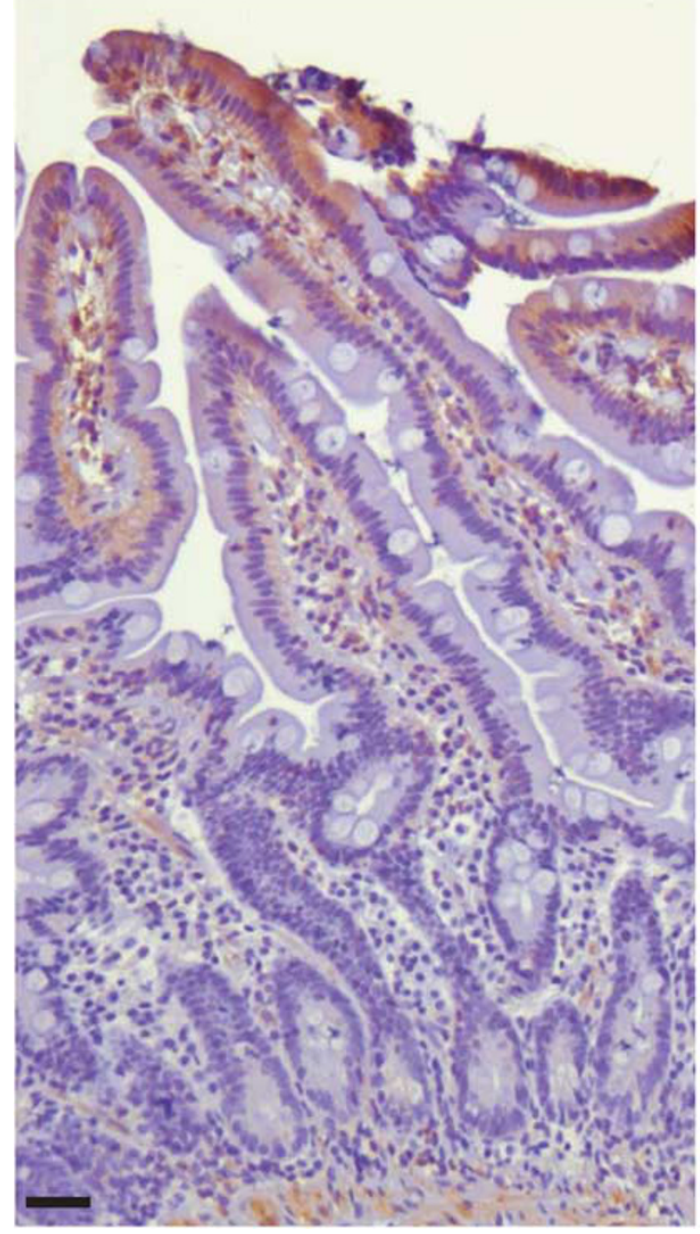

b

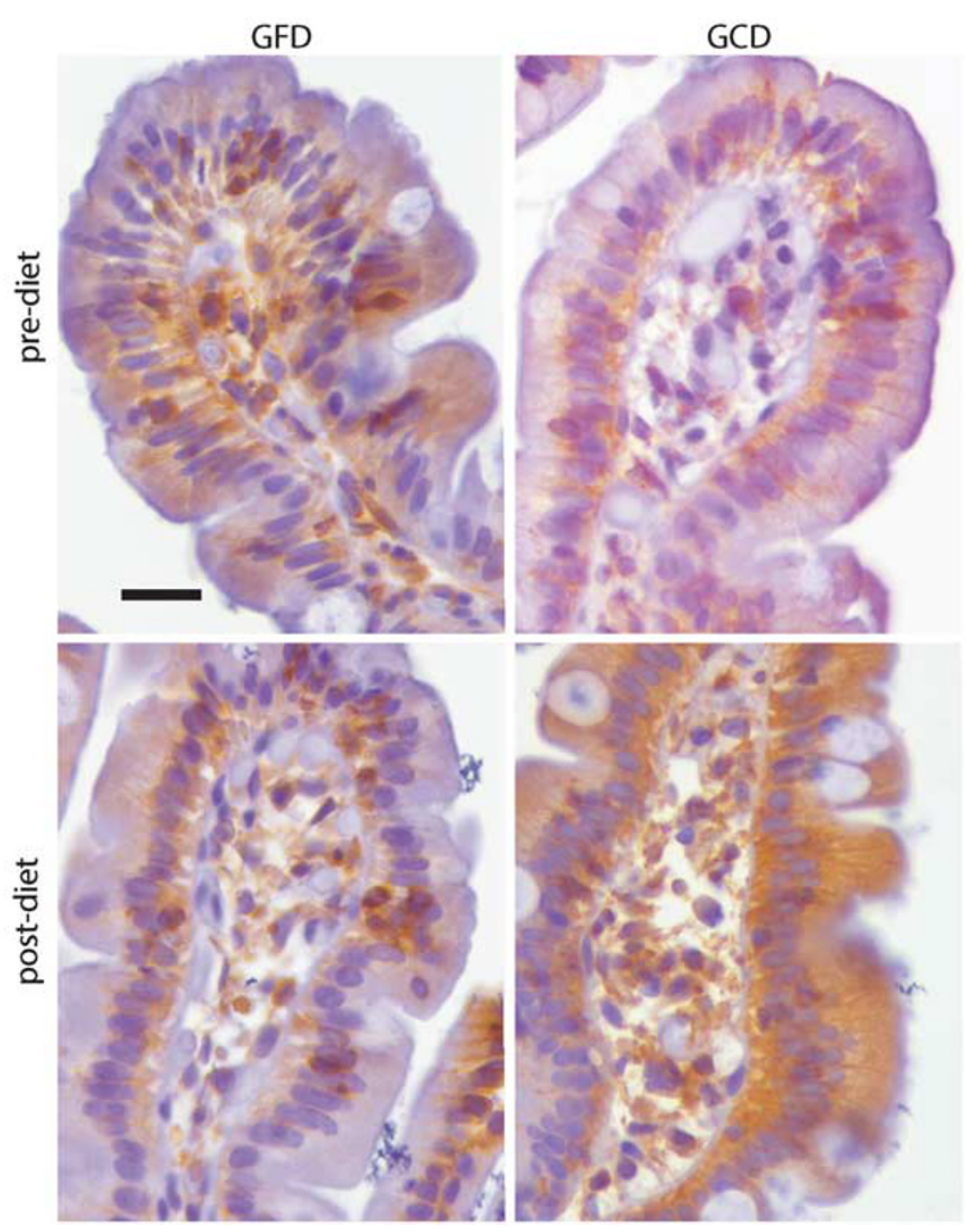

Figure 1 Myosin II regulatory light chain (MLC) phosphorylation increases after gluten-containing diet (GCD) challenge and correlates with increased small intestinal permeability. (a) MLC phosphorylation is most prominent in villus enterocytes. Bar $=50 \mu \mathrm{m}$. (b) Immunostains showing decreased or increased MLC phosphorylation in small intestinal mucosae following challenge with gluten-free diet (GFD) or GCD, respectively. MLC phosphorylation within small intestinal epithelium was similar before dietary challenge. $B a r=25 \mu \mathrm{m}$.

claudin-8 expression in this study group. Studies in knockout mice have shown that claudin-15, which forms a paracellular cation channel, can compensate for loss of claudin-2 expression. ${ }^{12,16,39}$ Despite these and other data indicating that function of claudin-15 is important, ${ }^{13,40-43}$ it has not been previously assessed in human disease. We therefore focused our analysis on claudins 2, 8, and 15 (Supplementary Figures 1 and 2).

\section{Claudin-2}

The paracellular cation and water channel claudin-2 was primarily detected at cell membranes at sites corresponding to the apical junctional complex. Faint cytoplasmic staining was also observed. In all biopsies, expression was greatest in crypt regions, consistent with previous studies of claudin-2 expression in rodent and human intestinal mucosae. ${ }^{37,44}$ Claudin-2 expression in small intestinal and colonic mucosae was not significantly affected by GFD or GCD, either when group expression data or individual changes were compared.

\section{Claudin-8}

Claudin-8 was detected within the cytoplasm and at apical cell junctions throughout the vertical axis. Neither small intestinal nor colonic claudin-8 expression was significantly affected by GFD or GCD. As with claudin-2, this was true whether group expression data or individual changes were analyzed.

\section{Claudin-15}

The paracellular cation channel claudin- 15 was present within the cytoplasm and at cell membranes near the apical junctional complex (Figure 2a). Expression was detected throughout the vertical axis, that is, from crypt to villus or surface epithelium. Small intestinal claudin-15 expression was not significantly affected by GCD or GFD. In contrast, colonic claudin-15 expression was markedly altered after dietary 
a

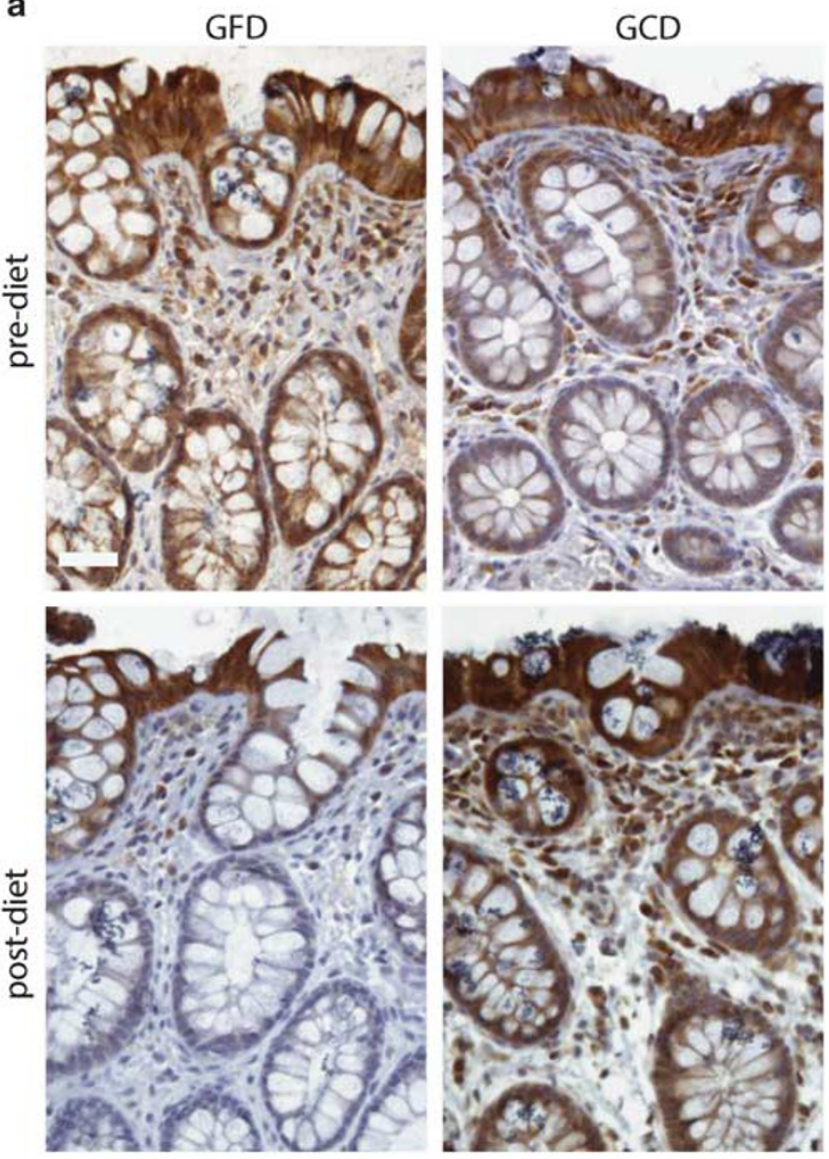

b

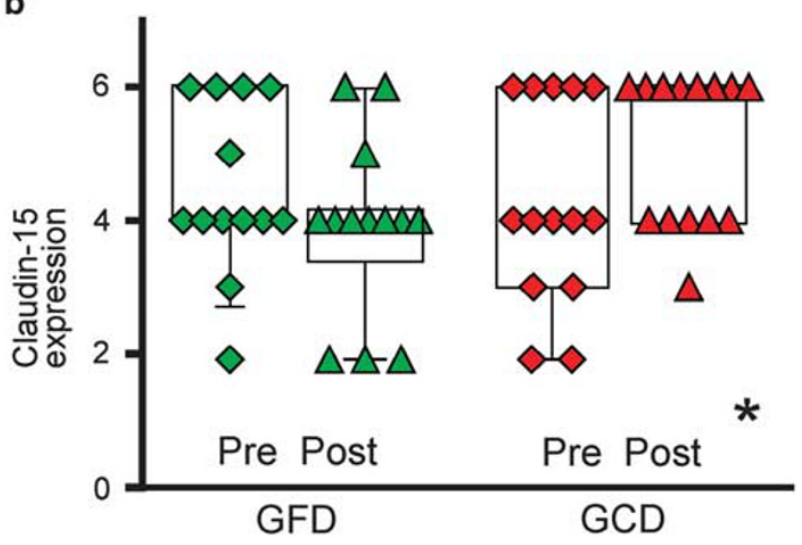

Figure 2 Claudin-15 expression increases after gluten-containing diet (GCD) challenge. (a) Immunostains showing claudin-15 expression in colonic mucosae before and after challenge with gluten-free diet (GFD) or $G C D$. Bar $=25 \mu \mathrm{m}$. (b) For subjects challenged with $G C D$, there was a significant increase in claudin-15 expression $\left({ }^{*} P<0.05\right)$.

gluten challenge (Figure $2 \mathrm{~b} ; \mathrm{P}<0.05$ ), with decreased and increased claudin-15 expression after GFD and GCD, respectively.

\section{Functional Analyses}

To better assess the relationships between immunohistochemical markers of intestinal barrier function and measured

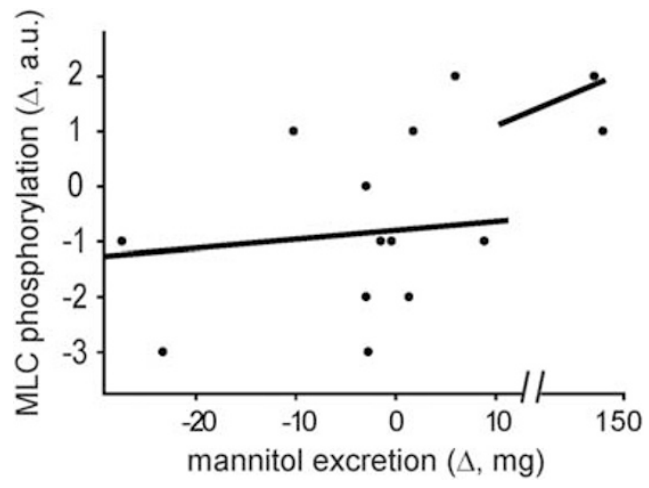

Figure 3 Increased myosin II regulatory light chain (MLC) phosphorylation correlates directly with intestinal permeability following gluten-containing diet (GCD). For subjects challenged with GCD, there was a direct correlation between the changes in MLC phosphorylation and mannitol excretion, a measure of intestinal permeability $(R=0.54$, $P<0.03)$.

small intestinal barrier function, we correlated changes in staining parameters with changes in urinary mannitol recovery. We focused on small intestinal barrier function because previous work has most consistently linked this, rather than colonic barrier function, to IBS-D. ${ }^{5}$ Importantly, mannitol is small and likely to reflect changes in tight junction pore pathway permeability rather than epithelial damage. ${ }^{45}$

As with previous analyses, mannitol recovery varied widely within and between groups. ${ }^{5,19}$ It is, therefore, not surprising that neither GFD nor GCD impacted intestinal permeability, measured as urinary mannitol recovery, when group data were compared. However, when changes in urinary mannitol recovery were assessed, GFD decreased and GCD increased recovery by an average of 2.7 and $16.4 \mathrm{mg}$, respectively. Nevertheless, a high degree of inter-individual variability prevented this from achieving statistical significance $(P=0.07)$.

To more directly probe a potential link between GCD, MLC phosphorylation, and increased urinary mannitol recovery, the GCD-induced changes in each of these parameters were correlated. In GCD-challenged subjects, increased small intestinal epithelial MLC phosphorylation correlated positively with increased mannitol excretion (Figure 3; $R=0.54, P<0.03$ ).

Claudin-2 is well recognized as forming a paracellular channel that transports small cations, that is, $\mathrm{Na}^{+}$, and water. ${ }^{28,46,47}$ It is less well recognized that claudin-2- and claudin-15-mediated efflux of $\mathrm{Na}^{+}$from the lamina propria to the lumen allows the $\mathrm{Na}^{+}$recycling that is necessary to support $\mathrm{Na}^{+}$-dependent ion and nutrient transport. Thus, perhaps counterintuitively, increased claudin- 2 or claudin- 15 expression can increase intestinal absorption of water and nutrients. ${ }^{12,16,45,47,48}$ Consistent with this, increased colonic claudin-2 expression, particularly within crypts, was 
a
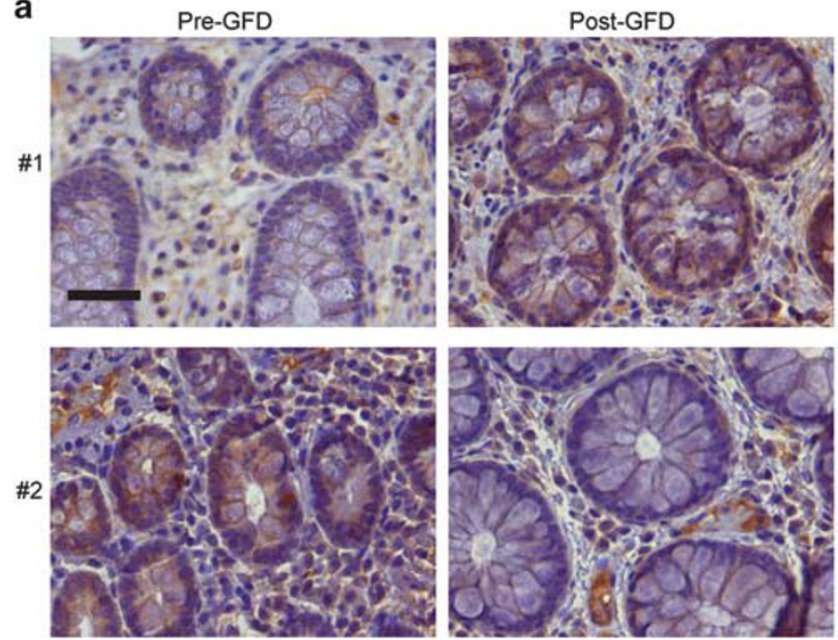

b

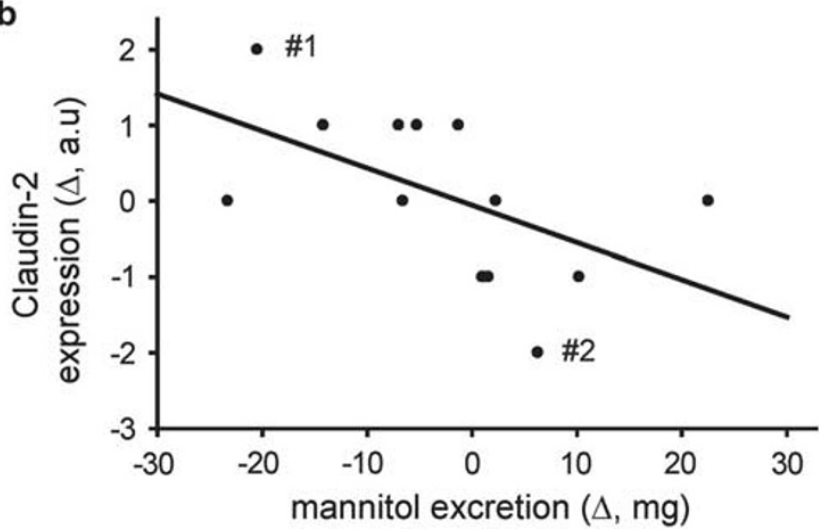

Figure 4 Changes in claudin-2 expression correlate inversely with intestinal permeability. (a) Immunostains showing claudin-2 expression in colonic mucosae from two different subjects $(\# 1, \# 2)$ before and after challenge with gluten-free diet (GFD). Bar $=25 \mu \mathrm{m}$. (b) There was an inverse correlation between claudin-2 expression and mannitol excretion $(R=0.55, P<0.03)$. Points indicating the two subjects shown in (a) are labeled.

significantly associated with decreased urinary mannitol recovery in subjects receiving a GFD (Figure 4; $R=0.55$, $P<0.03$ ). Correlations between changes in claudin- 8 or claudin-15 expression were small or not significantly correlated with urinary mannitol recovery.

\section{DISCUSSION}

This study follows a previous clinical trial of GFD in patients with IBS-D. ${ }^{18}$ In that study, challenge with GCD resulted in greater numbers of bowel movements and increased small intestinal permeability, as assessed by urinary mannitol recovery, relative to subjects challenged with GFD. Analysis of biopsies taken before and after dietary challenge failed to detect changes in mucosal histology, including numbers of intraepithelial lymphocytes, intramucosal mast cells or epithelial damage. ${ }^{18}$ Thus, paracellular permeability, which is primarily a function of tight junction permeability, was considered the most likely explanation for increased mannitol flux. Consistent with this, in vitro analyses of cytokine production by peripheral blood mononuclear cells from the IBS-D patients studied here showed modest increases in IL-10, GM-CSF, and TNF expression in response to gluten, relative to rice. ${ }^{18}$

The previous clinical trial of GCD compared with GFD did detect modest changes in tight junction protein mRNA content within mucosal biopsies. ${ }^{18}$ It was not, however, clear if this reflected alterations in expression by epithelial or other cell types within the mucosa. For example, intraepithelial $\gamma \delta \mathrm{T}$ lymphocytes have been shown to express occludin, which regulates their intraepithelial migration. ${ }^{49,50}$ Although small changes observed may be meaningful, immunohistochemical analyses failed to demonstrate changes in epithelial ZO-1 expression or distribution. ${ }^{18}$ Other studies have reported reduced claudin-1 protein and mRNA expression in IBS-D patients, relative to healthy controls, ${ }^{51}$ that are consistent with the increased claudin-1 mRNA expression following GFD in the patients studied here. ${ }^{18}$ However, neither expression nor distribution of occludin or claudin-1 proteins were analyzed in response to GCD or GFD challenge. ${ }^{18}$ Further, other studies have described increased claudin-1 expression in IBS with constipation (IBS-C), ${ }^{52}$ which we have recently found to lack changes in paracellular permeability (submitted for publication, Grover et al). Thus, although mucosal claudin-1 expression is likely reduced in IBS-D patients relative to healthy controls, as well as following challenge with GCD, relative to challenge with GFD, the impact on paracellular permeability remains unknown. The relevance of the small changes in claudin-1 expression observed is also called into question by the lack of reports suggesting any effect of an $\sim 30 \%$ reduction in claudin-1 expression, although complete knockout or nearly complete knockdown of claudin-1 expression has been associated with defective epidermal barrier function in mice ${ }^{53}$ and reduced intestinal epithelial barrier function in vitro. ${ }^{54}$ Further, given the low level of claudin-1 expression within the intestine $e^{44}$ and other studies that failed to detect altered claudin-1 expression in IBS-D patients, ${ }^{55}$ it is unlikely that changes in claudin-1 expression can explain barrier loss associated with IBS-D. Rather, reduced claudin-1 expression may be a marker of overall epithelial health, as it can be restored by ex vivo culture with glutamine. ${ }^{56}$ Similarly, modest reductions in occludin and ZO-1 expression are also unlikely to explain observed permeability changes given the normal intestinal barrier function of occludin knockout mice ${ }^{8,9}$ and the functional redundancy between ZO-1 and ZO-2.57,58 Thus, although many studies have confirmed the presence of intestinal barrier defects in IBS-D patients, ${ }^{25,51,59}$ the mechanisms underlying such barrier loss remain undefined.

To better characterize the mechanisms responsible for intestinal permeability changes in IBS-D patients, we appraised biopsies from the carefully characterized group that participated in a previous clinical trial assessing responses 
to GCD versus GFD. This study design has the advantage that all subjects were IBS-D patients and that there was a direct, timed intervention with biopsies before and after the intervention. In addition, subjects were carefully screened to exclude celiac disease, as well as other potentially confounding factors, for example, use of nonsteroidal anti-inflammatory drugs. ${ }^{18}$ Challenge with GFD provided benefit relative to challenge with GCD, as measured by clinical features and laboratory data. We therefore considered this to be an ideal study set that provided a unique opportunity to examine tight junction protein expression and distribution as well as intestinal permeability in a site-specific manner. Notably, no patients were on GFD, that is, they were all actively ingesting gluten, before the beginning of the study and during the predietary intervention stage. One may, therefore, ask how they were affected by GCD. The likely explanation is that their gluten intake was intermittent and variable, as expected in a normal diet, during the pre-dietary intervention part of the trial. In contrast, the GCD challenge provided gluten with each meal and likely resulted in greater gluten exposure than the patients' normal diets.

The use of paired biopsies before and after experimental dietary intervention allowed us to study tight junction protein expression as a function of diet without confounding factors such as inter-individual variability. This approach was particularly powerful because diet affected both intestinal permeability and bowel movement frequency. Further, all subjects were IBS-D patients, thereby eliminating concerns regarding other potential differences between healthy subjects and patients. Finally, the randomization of a single group of IBS-D patients to either GFD or GCD arms provides analytical power that is unavailable in comparisons between significantly different populations, for example, healthy and IBS-D subjects. Importantly, permeability changes detected were quantitatively small relative to changes reported in IBD, consistent with the absence of frank epithelial damage in the patients studied here.

Our observation that no anatomic disease was detectable on traditional hematoxylin and eosin-stained sections confirms many previous studies and also demonstrates that permeability changes measured here cannot be secondary to epithelial damage, that is, the unrestricted flux pathway. ${ }^{60} \mathrm{We}$ therefore focused on the primary known regulators of tight junction barrier function. MLCK-dependent MLC phosphorylation is now a well-established regulator of tight junction permeability in response to both physiological ${ }^{61-65}$ and pathophysiological ${ }^{17,20,24}$ stimuli, in both in vitro and in vivo experimental models, as well as excised human tissue. Increases in tight junction permeability triggered by MLC phosphorylation result in enhanced paracellular flux. ${ }^{62,65-67}$ As phosphorylated MLC content reflects the balance between kinase and phosphatase activities as well as transcriptional regulation of MLCK expression, ${ }^{20,68,69}$ we assessed both MLCK expression and MLC phosphorylation in each biopsy.
Small intestinal MLC phosphorylation tended to decrease after challenge with GFD and increase after GCD diet. Neither change reached statistical significance, likely due to the relatively small sample size of this invasive study. Nevertheless, the changes were reciprocal and correlated significantly with mannitol excretion at $2-8 \mathrm{~h}$, that is, small intestinal permeability. These results provide evidence that this mechanism of tight junction regulation is activated during symptomatic IBS-D and downregulated during symptom abatement. Notably, MLCK expression and activation have been shown to be exquisitely sensitive to TNF, ${ }^{7,24,38,64,68-70}$ consistent with previous in vitro analyses of peripheral blood mononuclear cells from the IBS-D patients studied here that demonstrated a trend toward increased TNF expression in response to gluten, relative to rice. ${ }^{18}$ Such MLCK activation is well recognized to drive occludin endocytosis, ${ }^{7,24}$ which unifies our observations with previous work that found increases in MLC phosphorylation and cytoplasmic occludin staining in IBS-D patients, relative to healthy control subjects. ${ }^{55}$

To understand the relevance of altered claudin protein expression profiles, it is essential to recognize that intestinal paracellular permeability reflects the integrated function of the ensemble of claudin proteins expressed. As noted above, modest changes in claudin-1 expression are unlikely to critically impact intestinal barrier function. Rather, we focused on claudin-2, claudin-15 and claudin-8. Claudin-2 forms a paracellular $\mathrm{Na}^{+}$and water channel $28,29,71$ that increases cation flux across the highly selective, high capacity pore pathway. ${ }^{72,73}$ Claudin-2 is typically expressed at low levels after the neonatal period, ${ }^{44}$ but is upregulated in inflammation-associated intestinal diseases, including IBD, and in response to MLCK-driven barrier loss. ${ }^{21,36-38,74}$ Claudin-8 has been reported to act as an inhibitor of claudin-2 channel function, thereby reducing intestinal permeability. ${ }^{30}$ Like claudin-2, claudin-15 forms a paracellular $\mathrm{Na}^{+}$channel that enhances pore pathway flux. Notably, claudin-15 is expressed in a pattern that complements that of claudin-2, that is, intestinal epithelial claudin-15 expression increases after the neonatal period. ${ }^{44}$ These and other data ${ }^{12,16}$ indicate that claudin- 2 and claudin-15 are at least partially redundant in terms of function despite markedly different expression patterns during development and disease.

When considered in the context of the functional impact of MLC phosphorylation and claudin-2, 8 and 15 expression, our data can be unified to provide a potential mechanism for increased intestinal permeability and bowel movement number in IBS, as represented by the GCD group, relative to health, that is, the GFD group (Figure 5). In those challenged with a GCD, we detected increased small intestinal MLC phosphorylation, which is known to enhance paracellular water efflux. ${ }^{17,24,72}$ Increased colonic claudin-15 expression would be expected to compound this problem by allowing $\mathrm{Na}^{+}$efflux. However, this could also be seen as an adaptive response that enhances nutrient and water 

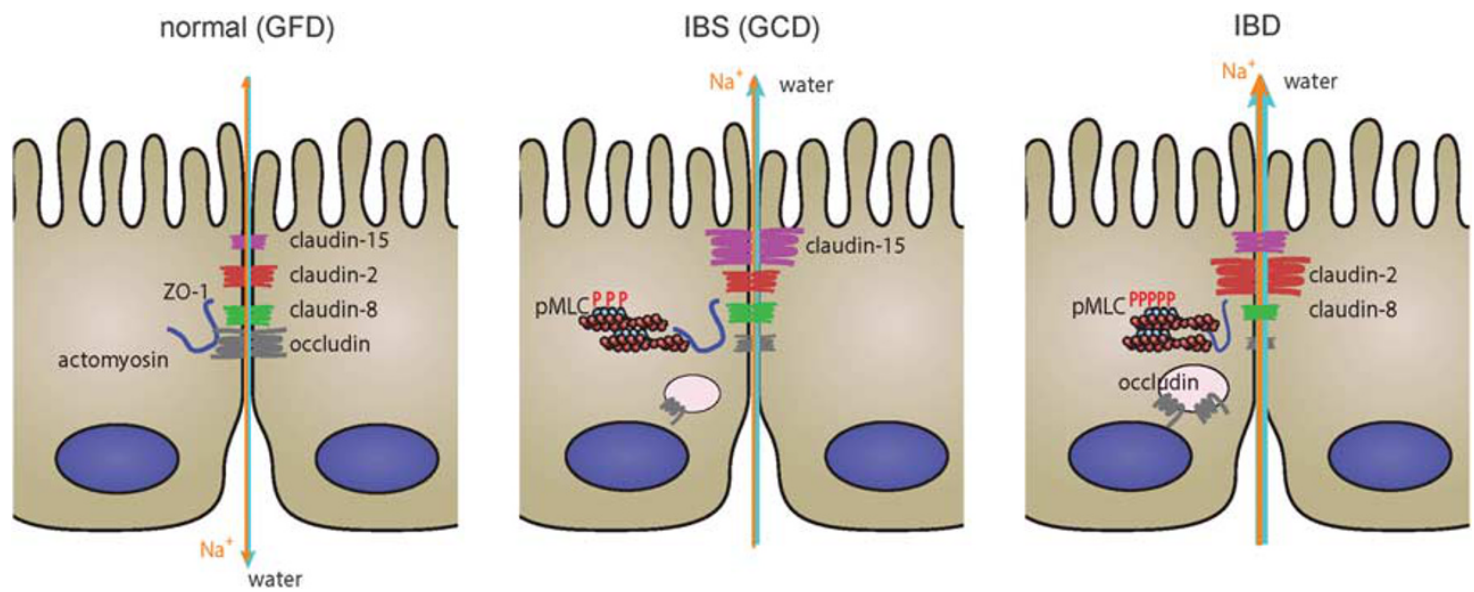

Figure 5 Comparison of alterations in tight junction regulation and net transepithelial transport in health, irritable bowel syndrome (IBS) and inflammatory bowel disease (IBD). It is possible to explain the pathophysiology observed in IBS and why it differs from that in IBD on the basis of the data presented here and published data.

absorption by allowing paracellular $\mathrm{Na}^{+}$efflux that supports further $\mathrm{Na}^{+}$-dependent transcellular transport. Similarly, the correlation between increased colonic claudin-2 expression and reduced intestinal permeability in those challenged with GFD could reflect the ability of claudin-2 to promote mucosal to luminal $\mathrm{Na}^{+}$efflux across crypt epithelium in order to support the osmotic gradient that drives luminal to mucosal paracellular water absorption. ${ }^{75}$ Together with our demonstration that claudin-15 is expressed in crypt, as well as surface and villus epithelium, whereas claudin-2 is restricted to crypt epithelium, these observations may explain the partially contrasting changes in claudin-2 and claudin- 15 expression in response to dietary challenges. Finally, it is interesting to note previous studies showing reduced JAM-A expression in IBS-D patients ${ }^{76}$ and a separate report demonstrating increased claudin-15 expression in JAM-A knockout mice. ${ }^{77}$ In vitro studies further suggest that mast cell degranulation and tryptase release results in JAM-A and claudin-1 downregulation. ${ }^{76}$ These data support the hypothesis that mast cell activity may be responsible for some intestinal permeability increases associated with IBS-D, and this topic is deserving of further study.

The changes seen after GCD challenge in IBS-D are similar to, although distinct from, changes seen in IBD. ${ }^{20,36,37}$ Both are associated with increased MLC phosphorylation and increased expression of a cation pore-forming claudin (Figure 5), although some data suggest that single-channel conductances across claudin- 2 channels ${ }^{29}$ may be greater than those across claudin-15 channels.

We also prevent evidence that, similar to IBD, conductance across the paracellular macromolecular flux pathway is increased in IBS-D as a result of increased MLC phosphorylation. However, because macromolecular barrier defects associated with IBS-D are typically less extensive than those seen in IBD, we hypothesize that the MLC phosphorylation in
IBS-D may be less than that occurring in IBD and closer to that associated with $\mathrm{Na}^{+}$-glucose cotransport. ${ }^{65}$ Nevertheless, it will be interesting to determine if increased MLC phosphorylation in IBS-D is associated with TNF polymorphisms that have also been linked to IBS-D, ${ }^{78}$ as MLCK is activated by TNF. $24,64,68$

In conclusion, these data provide evidence that two wellestablished mechanisms of increasing paracellular permeability, that is, reducing tight junction barrier function, by distinct pathways are activated by gluten challenge in IBD patients. Whether other interventions that correct barrier loss will have therapeutic benefit remains an important question for future studies.

Supplementary Information accompanies the paper on the Laboratory Investigation website (http://www.laboratoryinvestigation.org)

\section{ACKNOWLEDGMENTS}

This study was supported by National Institute of Health Grants R01DK61931 (JRT); R01DK68271 (JRT); R24DK099803 (JRT); and R01DK092179 (MC).

\section{DISCLOSURE/CONFLICT OF INTEREST}

The authors declare no conflict of interest.

1. Drossman DA, Chang L, Bellamy $\mathrm{N}$, et al. Severity in irritable bowel syndrome: a Rome Foundation Working Team report. Am J Gastroenterol 2011;106:1749-1759; quiz 1760.

2. Sood R, Law GR, Ford AC. Diagnosis of IBS: symptoms, symptom-based criteria, biomarkers or 'psychomarkers'? Nat Rev Gastroenterol Hepatol 2014;11:683-691.

3. Peery AF, Dellon ES, Lund J, et al. Burden of gastrointestinal disease in the United States: 2012 update. Gastroenterol 2012;143: 1179-1187.e1173.

4. Camilleri M, Katzka DA. Irritable bowel syndrome: methods, mechanisms, and pathophysiology. Genetic epidemiology and pharmacogenetics in irritable bowel syndrome. Am J Physiol Gastrointest Liver Physiol 2012;302:G1075-G1084.

5. Vazquez-Roque MI, Camilleri M, Smyrk T, et al. Association of HLA-DQ gene with bowel transit, barrier function, and inflammation in irritable bowel syndrome with diarrhea. Am J Physiol Gastrointest Liver Physiol 2012;303:G1262-G1269. 
6. Camilleri M, Madsen $\mathrm{K}$, Spiller $\mathrm{R}$, et al. Intestinal barrier function in health and gastrointestinal disease. Neurogastroenterol Motil 2012;24: 503-512.

7. Marchiando AM, Shen L, Graham WV, et al. Caveolin-1-dependent occludin endocytosis is required for TNF-induced tight junction regulation in vivo. J Cell Biol 2010;189:111-126.

8. Saitou M, Furuse M, Sasaki H, et al. Complex phenotype of mice lacking occludin, a component of tight junction strands. Mol Biol Cell 2000;11: 4131-4142.

9. Schulzke JD, Gitter AH, Mankertz J, et al. Epithelial transport and barrier function in occludin-deficient mice. Biochim Biophys Acta 2005;1669: 34-42.

10. Marchiando AM, Graham WV, Turner JR. Epithelial barriers in homeostasis and disease. Annu Rev Pathol 2010;5:119-144.

11. Muto S, Hata M, Taniguchi J, et al. Claudin-2-deficient mice are defective in the leaky and cation-selective paracellular permeability properties of renal proximal tubules. Proc Natl Acad Sci USA 2010;107: 8011-8016.

12. Tamura A, Hayashi $H$, Imasato $M$, et al. Loss of claudin-15, but not claudin-2, causes $\mathrm{Na}+$ deficiency and glucose malabsorption in mouse small intestine. Gastroenterol 2011;140:913-923.

13. Tamura A, Kitano $\mathrm{Y}$, Hata $\mathrm{M}$, et al. Megaintestine in claudin-15deficient mice. Gastroenterol 2008;134:523-534.

14. Bagherie-Lachidan M, Wright SI, Kelly SP. Claudin-8 and -27 tight junction proteins in puffer fish Tetraodon nigroviridis acclimated to freshwater and seawater. J Comp Physiol B 2009;179: 419-431.

15. Hou J, Renigunta A, Yang J, et al. Claudin-4 forms paracellular chloride channel in the kidney and requires claudin-8 for tight junction localization. Proc Natl Acad Sci USA 2010;107:18010-18015.

16. Wada M, Tamura A, Takahashi N, et al. Loss of claudins 2 and 15 from mice causes defects in paracellular $\mathrm{Na}+$ flow and nutrient transport in gut and leads to death from malnutrition. Gastroenterol 2013;144: 369-380.

17. Clayburgh DR, Musch MW, Leitges M, et al. Coordinated epithelial NHE3 inhibition and barrier dysfunction are required for TNF-mediated diarrhea in vivo. J Clin Invest 2006;116:2682-2694.

18. Vazquez-Roque MI, Camilleri M, Smyrk T, et al. A controlled trial of gluten-free diet in patients with irritable bowel syndrome-diarrhea: effects on bowel frequency and intestinal function. Gastroenterol 2013;144:903-911 e903.

19. Rao AS, Camilleri M, Eckert DJ, et al. Urine sugars for in vivo gut permeability: validation and comparisons in irritable bowel syndromediarrhea and controls. Am J Physiol Gastrointest Liver Physiol 2011;301: G919-G928.

20. Blair SA, Kane SV, Clayburgh DR, et al. Epithelial myosin light chain kinase expression and activity are upregulated in inflammatory bowel disease. Lab Invest 2006;86:191-201.

21. Weber CR, Nalle SC, Tretiakova $M$, et al. Claudin-1 and claudin-2 expression is elevated in inflammatory bowel disease and may contribute to early neoplastic transformation. Lab Invest 2008;88:1110-1120.

22. Russo JM, Florian P, Shen L, et al. Distinct temporal-spatial roles for rho kinase and myosin light chain kinase in epithelial purse-string wound closure. Gastroenterol 2005;128:987-1001.

23. Clayburgh DR, Rosen S, Witkowski ED, et al. A differentiationdependent splice variant of myosin light chain kinase, MLCK1, regulates epithelial tight junction permeability. J Biol Chem 2004;279: 55506-55513.

24. Clayburgh DR, Barrett TA, Tang Y, et al. Epithelial myosin light chain kinase-dependent barrier dysfunction mediates $T$ cell activationinduced diarrhea in vivo. J Clin Invest 2005;115:2702-2715.

25. Dunlop SP, Hebden J, Campbell E, et al. Abnormal intestinal permeability in subgroups of diarrhea-predominant irritable bowel syndromes. Am J Gastroenterol 2006;101:1288-1294.

26. Van Itallie CM, Holmes J, Bridges A, et al. The density of small tight junction pores varies among cell types and is increased by expression of claudin-2. J Cell Sci 2008;121:298-305.

27. Li J, Zhuo M, Pei $\mathrm{L}$, et al. Comprehensive cysteine-scanning mutagenesis reveals claudin-2 pore-lining residues with different intrapore locations. J Biol Chem 2014;289:6475-6484.

28. $\mathrm{Yu}$ AS, Cheng $\mathrm{MH}$, Angelow $\mathrm{S}$, et al. Molecular basis for cation selectivity in claudin-2-based paracellular pores: identification of an electrostatic interaction site. J Gen Physiol 2009;133:111-127.
29. Weber CR, Liang GH, Wang Y, et al. Claudin-2-dependent paracellular channels are dynamically gated. eLife 2015;4:e09906.

30. Angelow S, Schneeberger EE, Yu AS. Claudin-8 expression in renal epithelial cells augments the paracellular barrier by replacing endogenous claudin-2. J Membr Biol 2007;215:147-159.

31. Angelow S, Kim KJ, Yu AS. Claudin-8 modulates paracellular permeability to acidic and basic ions in MDCK II cells. J Physiol 2006;571: 15-26.

32. Yu AS, Enck AH, Lencer WI, et al. Claudin-8 expression in Madin-Darby canine kidney cells augments the paracellular barrier to cation permeation. J Biol Chem 2003;278:17350-17359.

33. Colegio OR, Van Itallie C, Rahner C, et al. Claudin extracellular domains determine paracellular charge selectivity and resistance but not tight junction fibril architecture. Am J Physiol Cell Physiol 2003;284: C1346-C1354.

34. Capaldo CT, Farkas AE, Hilgarth RS, et al. Proinflammatory cytokineinduced tight junction remodeling through dynamic self-assembly of claudins. Mol Biol Cell 2014;25:2710-2719.

35. Szakal DN, Gyorffy $H$, Arato A, et al. Mucosal expression of claudins 2, 3 and 4 in proximal and distal part of duodenum in children with coeliac disease. Virchows Arch 2010;456:245-250.

36. Zeissig S, Burgel N, Gunzel D, et al. Changes in expression and distribution of claudin 2, 5 and 8 lead to discontinuous tight junctions and barrier dysfunction in active Crohn's disease. Gut 2007;56:61-72.

37. Heller F, Florian P, Bojarski C, et al. Interleukin-13 is the key effector Th2 cytokine in ulcerative colitis that affects epithelial tight junctions, apoptosis, and cell restitution. Gastroenterol 2005;129:550-564.

38. Su L, Nalle SC, Shen L, et al. TNFR2 activates MLCK-dependent tight junction dysregulation to cause apoptosis-mediated barrier loss and experimental colitis. Gastroenterol 2013;145:407-415.

39. Suzuki H, Nishizawa T, Tani K, et al. Crystal structure of a claudin provides insight into the architecture of tight junctions. Science 2014;344:304-307.

40. Abiko $Y$, Kojima $T$, Murata $M$, et al. Changes of tight junction protein claudins in small intestine and kidney tissues of mice fed a DDC diet. J Toxicol Pathol 2013;26:433-438.

41. Arimura $Y$, Nagaishi K, Hosokawa M. Dynamics of claudins expression in colitis and colitis-associated cancer in rat. Methods Mol Biol 2011;762:409-425.

42. Darsigny $M$, Babeu JP, Dupuis $A A$, et al. Loss of hepatocyte-nuclearfactor-4alpha affects colonic ion transport and causes chronic inflammation resembling inflammatory bowel disease in mice. PLoS One 2009;4:e7609.

43. Tipsmark CK, Sorensen KJ, Madsen SS. Aquaporin expression dynamics in osmoregulatory tissues of Atlantic salmon during smoltification and seawater acclimation. J Exp Biol 2010;213:368-379.

44. Holmes JL, Van Itallie CM, Rasmussen JE, et al. Claudin profiling in the mouse during postnatal intestinal development and along the gastrointestinal tract reveals complex expression patterns. Gene Expr Patterns 2006;6:581-588.

45. Turner JR, Cohen DE, Mrsny RJ, et al. Noninvasive in vivo analysis of human small intestinal paracellular absorption: regulation by $\mathrm{Na}$ +-glucose cotransport. Dig Dis Sci 2000;45:2122-2126.

46. Rosenthal R, Gunzel D, Krug SM, et al. Claudin-2-mediated cation and water transport share a common pore. Acta Physiol (Oxf) 2016; doi:10.1111/apha.12742.

47. Amasheh S, Meiri N, Gitter AH, et al. Claudin-2 expression induces cation-selective channels in tight junctions of epithelial cells. J Cell Sci 2002;115:4969-4976.

48. Turner JR, Buschmann MM, Romero-Calvo I, et al. The role of molecular remodeling in differential regulation of tight junction permeability. Semin Cell Dev Biol 2014;36:204-212.

49. Edelblum $\mathrm{KL}$, Shen $\mathrm{L}$, Weber $\mathrm{CR}$, et al. Dynamic migration of gammadelta intraepithelial lymphocytes requires occludin. Proc Natl Acad Sci USA 2012;109:7097-7102.

50. Edelblum KL, Singh G, Odenwald MA, et al. Gammadelta intraepithelial lymphocyte migration limits transepithelial pathogen invasion and systemic disease in mice. Gastroenterol 2015;148:1417-1426.

51. Bertiaux-Vandaele N, Youmba SB, Belmonte L, et al. The expression and the cellular distribution of the tight junction proteins are altered in irritable bowel syndrome patients with differences according to the disease subtype. Am J Gastroenterol 2011;106:2165-2173. 
52. Cheng P, Yao J, Wang C, et al. Molecular and cellular mechanisms of tight junction dysfunction in the irritable bowel syndrome. Mol Med Rep 2015;12:3257-3264.

53. Furuse $M$, Hata $M$, Furuse $K$, et al. Claudin-based tight junctions are crucial for the mammalian epidermal barrier: a lesson from claudin-1deficient mice. J Cell Biol 2002;156:1099-1111.

54. Saeedi BJ, Kao DJ, Kitzenberg DA, et al. HIF-dependent regulation of claudin-1 is central to intestinal epithelial tight junction integrity. Mol Biol Cell 2015;26:2252-2262.

55. Martinez C, Lobo B, Pigrau M, et al. Diarrhoea-predominant irritable bowel syndrome: an organic disorder with structural abnormalities in the jejunal epithelial barrier. Gut 2013;62:1160-1168.

56. Bertrand J, Ghouzali I, Guerin C, et al. Glutamine restores tight junction protein claudin-1 expression in colonic mucosa of patients with diarrhea-predominant irritable bowel syndrome. J Parenter Enteral Nutr 2015; doi:10.1177/0148607115587330.

57. Umeda K, Ikenouchi J, Katahira-Tayama S, et al. ZO-1 and ZO-2 independently determine where claudins are polymerized in tight-junction strand formation. Cell 2006;126:741-754.

58. Wilcz-Villega E, McClean S, O'Sullivan M. Reduced E-cadherin expression is associated with abdominal pain and symptom duration in a study of alternating and diarrhea predominant IBS. Neurogastroenterol Motil 2014;26:316-325.

59. Piche $T$, Barbara $G$, Aubert $P$, et al. Impaired intestinal barrier integrity in the colon of patients with irritable bowel syndrome: involvement of soluble mediators. Gut 2009;58:196-201.

60. Nalle SC, Turner JR. Intestinal barrier loss as a critical pathogenic link between inflammatory bowel disease and graft-versus-host disease. Mucosal Immunol 2015;8:720-730.

61. Turner JR, Madara JL. Physiological regulation of tight junction permeability by $\mathrm{Na}$ +-nutrient cotransport. In: Anderson JM, Cereijido M (eds). Tight Junctions: Academic Press: Boca Raton, FL, USA, 2001, p 333-347.

62. Turner JR, Rill BK, Carlson SL, et al. Physiological regulation of epithelial tight junctions is associated with myosin light-chain phosphorylation. Am J Physiol-Cell Physiol 1997;273:C1378-C1385.

63. Atisook K, Carlson S, Madara JL. Effects of phlorizin and sodium on glucose-elicited alterations of cell junctions in intestinal epithelia. Am J Physiol 1990;258:C77-C85.

64. Zolotarevsky Y, Hecht G, Koutsouris A, et al. A membrane-permeant peptide that inhibits MLC kinase restores barrier function in in vitro models of intestinal disease. Gastroenterol 2002;123:163-172.

65. Berglund JJ, Riegler M, Zolotarevsky Y, et al. Regulation of human jejunal transmucosal resistance and MLC phosphorylation by
$\mathrm{Na}^{+}$-glucose cotransport. Am J Physiol Gastrointest Liver Physiol 2001;281:G1487-G1493.

66. Shen L, Black ED, Witkowski ED, et al. Myosin light chain phosphorylation regulates barrier function by remodeling tight junction structure. J Cell Sci 2006;119:2095-2106.

67. Su L, Shen L, Clayburgh DR, et al. Targeted epithelial tight junction dysfunction causes immune activation and contributes to development of experimental colitis. Gastroenterol 2009;136:551-563.

68. Wang F, Graham WV, Wang $Y$, et al. Interferon-gamma and tumor necrosis factor-alpha synergize to induce intestinal epithelial barrier dysfunction by up-regulating myosin light chain kinase expression. Am J Pathol 2005;166:409-419.

69. Graham WV, Wang F, Clayburgh DR, et al. Tumor necrosis factorinduced long myosin light chain kinase transcription is regulated by differentiation-dependent signaling events. Characterization of the human long myosin light chain kinase promoter. J Biol Chem 2006;281: 26205-26215.

70. Wang F, Schwarz BT, Graham WV, et al. IFN-gamma-induced TNFR2 expression is required for TNF-dependent intestinal epithelial barrier dysfunction. Gastroenterol 2006;131:1153-1163.

71. Rosenthal R, Milatz S, Krug SM, et al. Claudin-2, a component of the tight junction, forms a paracellular water channel. J Cell Sci 2010;123: 1913-1921.

72. Turner JR. Intestinal mucosal barrier function in health and disease. Nat Rev Immunol 2009;9:799-809.

73. Anderson JM, Van Itallie CM. Physiology and function of the tight junction. Cold Spring Harb Perspect Biol 2009;1:a002584.

74. Weber CR, Raleigh DR, Su L, et al. Epithelial myosin light chain kinase activation induces mucosal interleukin-13 expression to alter tight junction ion selectivity. J Biol Chem 2010;285:12037-12046.

75. Marciani L, Cox EF, Hoad CL, et al. Postprandial changes in small bowel water content in healthy subjects and patients with irritable bowel syndrome. Gastroenterol 2010;138:469-477, 477 e461.

76. Wilcz-Villega EM, McClean S, O'Sullivan MA. Mast cell tryptase reduces junctional adhesion molecule-A (JAM-A) expression in intestinal epithelial cells: implications for the mechanisms of barrier dysfunction in irritable bowel syndrome. Am J Gastroenterol 2013;108: 1140-1151.

77. Vetrano S, Rescigno M, Cera MR, et al. Unique role of junctional adhesion molecule-a in maintaining mucosal homeostasis in inflammatory bowel disease. Gastroenterol 2008;135:173-184.

78. Swan C, Duroudier NP, Campbell E, et al. Identifying and testing candidate genetic polymorphisms in the irritable bowel syndrome (IBS): association with TNFSF15 and TNFalpha. Gut 2013;62:985-994. 